\title{
Indice de salud arrecifal (ISA) en los arrecifes coralinos de Cayos Miskitos
}

\author{
Reef Health Index (RHI) in the Coral Reefs from Miskitos Cayos
}

\author{
Marcos Williamson Cuthbert ${ }^{1}$ \\ Donald Williams Smith ${ }^{2}$ \\ Osmar Arróliga ${ }^{3}$ \\ José Mendoza ${ }^{4}$ \\ Norving Torres ${ }^{5}$ \\ Oscar Flores ${ }^{6}$
}

\section{Resumen}

Los arrecifes de coral de Cayos Miskitos se localizan en la Región Autónoma de la Costa Caribe Norte de Nicaragua. Conforman un mosaico submarino de arrecifes marginales, arrecifes de parche, lejanas crestas arrecifales orientadas de norte a sur, lechos de pastos marinos, algas y jardines de octocorales. Los corales son una clase de colonia de animales que se relacionan con los hidrozoos, las medusas y las anémonas de mar. Se ha diagnosticado el estado de salud de estos arrecifes coralinos, tomando como bioindicadores el estado de las colonias de corales marinos, la cobertura de algas carnosas, la diversidad y abundancia de peces herbívoros y peces de interés comercial. El estudio es cuantitativo y la evaluación se realizó a través del método transeptos lineales. Para esto se realizaron muestreos a través de buceo en 18 arrecifes coralinos preseleccionados, con un esfuerzo de dos transeptos de 30 metros de longitud en cada uno de esos arrecifes. El 50\% de los arrecifes muestreados presentan de buena a muy buena la condición de salud; el 33\% presentan condición regular; mientras el 17\% se encuentran en condición de mal a crítico. En general los resultados son satisfactorios, ya que los arrecifes de coral en cayos miskitos mantienen la función y estructura, proporcionando altos valores de biodiversidad acuática que favorecen el trabajo y alimentación de las comunidades indígenas costeras, las que toda su vida ha sobrevivido de sus bondades. ¿cuáles son las propuestas de mejorar la autostenibilidad?

Palabras clave: Arrecifes coralinos; Indice de salud; Cayos Miskitos.

\section{Abstract}

The coral reefs from Miskito Cays are located in the Autonomous Region of the North Nicaraguan Caribbean Coast. They form an underwater mosaic of marginal reefs, patch reefs, distant reef ridges oriented from north to south, seagrass beds, seaweed and octocoral gardens. Corals are a kind of animals' colony that are related to hydrozoans, jellyfish and sea anemones. The health status of these coral reefs has been diagnosed, taking as bioindicators the status of marine coral colonies, the cover of fleshy algae, the diversity and abundance of herbivorous fish and fish of commercial interest. The study is quantitative

\footnotetext{
Msc. en gobernanza y desarrollo de identidad, director de IREMADES-CISA, URACCAN, tutor de investigación; e-mail: marcoswillni123@gmail.com; No ORCID: http://orcid.org/0000-0002-1887-1328

2 Licenciado en Biología marina, Técnico de IREMADES, URACCAN, No. ORCID: http://orcid.org/0000-0002-1931-577X; e-mail; ronaldw1981@yahoo.com

3 Licenciado en Ecología. Técnico FUNDAR.

4 Licenciado en Biología. Técnico FUNDAR.

5 Msc en Manejo de Vida Silvestre. Presidente FUNDAR.

6 Licenciado en Ecología. Técnico FUNDAR.
}

Recibido: 16/08/2019 - Aprobado: 30/08/2019 
and the evaluation was carried out through the linear transept method. To this end, sampling was carried out through diving in 18 preselected coral reefs, with an effort of two 30-meter long transepts in each of those reefs. $50 \%$ of the sampled reefs have a good to very good health condition; $33 \%$ have a regular condition; while $17 \%$ are in a condition of bad to critical. In general, the results are satisfactory, since the coral reefs in Miskito cays maintain the function and structure, providing high values of aquatic biodiversity that favor the work and food of the coastal indigenous communities, which all their lives have survived their benefits. However, what are the proposals to improve self-sustainability?

Keywords: Coral Reefs, Health Index, Miskitos Cayos.

\section{Introducción}

El sistema de arrecifes coralinos de Cayos Miskitos, se ubica en la Región Autónoma de la Costa Caribe Norte de Nicaragua. Este ecosistema es de importancia para la conservación y el desarrollo humano, de acuerdo al modelo de autosostenibilidad de vida que practican los pueblos indígenas de la Mosquita nicaragüense. Considerando esta realidad, la Universidad de las Regiones Autónomas de la Costa Caribe Nicaragüense (URACCAN), Instituto de Recursos Naturales Medio Ambiente y Desarrollo Sostenible (IREMADES) y la Unión Internacional para la Conservación de la Naturaleza (UICN), suscriben un convenio para desarrollar el proyecto: "Fortalecimiento de condiciones y capacidades de la población para la conservación y manejo sostenible de ecosistemas costeros de la Costa Caribe".

Mediante este proyecto se realizó evaluación ecológica de los ecosistemas marinos (arrecifes coralinos y pastos marinos) que, se encuentran en el área protegida Reserva Biológica Cayos Miskitos, con el fin de conocer su estado actual de conservación, considerando que el área de Cayos Miskitos fue azotado por el huracán Félix en el 2007, además las actividades de pesca son cada vez mayores, convirtiéndose en una gran presión para los arrecifes coralinos. Los sitios y transeptos muestreados fueron los mismos utilizados por Fonseca et al (2001).

\section{Revisión de literatura}

Los arrecifes de coral de Cayos Miskitos se encuentran en la Reserva Biológica de Cayos Miskitos, la que forma parte del sistema de áreas protegidas de Nicaragua, según decreto 43-91, siendo sus recursos a proteger bancos de coral, pastos submarinos, tortugas marinas y manatíes (MARENA, 1999).

Dada la importancia de los arrecifes de coral, para el mantenimiento de la biodiversidad acuática, que es base de la subsistencia de las comunidades indígenas costeras, se han realizado una serie de trabajos de investigación para conocer el estado de conservación de los Cayos Miskitos. Phillips et al. (1982), realizaron el primer estudio de algas y pastos marinos de Cayos Miskitos. Ogden y Gladfelter (1983) y Marshall (1994), también se estudiaron los pastos marinos en esta área.

Alevizon (1993) y Jameson (1996) hacen una descripción cualitativa de los arrecifes coralinos de Cayos Miskitos y sus recursos pesqueros. Hasta ahora, los arrecifes más estudiados del Caribe de América Central son los de Belice, Honduras, Costa Rica y Panamá (Cortés, 1997).

Fonseca et al. (2001), realizan una evaluación ecológica rápida del sistema arrecifal de Cayos Miskitos, con el objetivo de describir y diagnosticar el estado de salud de los distintos ambientes y organismos marinos. Reportan 39 especies de corales, 43,4\%) de cobertura de coral vivo promedio. Este autor indica que los arrecifes de coral de Cayos Miskitos se encuentran en buen estado de salud, por su alta cobertura de coral vivo, baja mortalidad y alta diversidad. 


\section{Materiales y métodos}

Para determinar la condición de salud de los 18 arrecifes coralinos muestreados en Cayos Miskitos, se realizó entre los días 03 al 11 de abril de 2018, una evaluación a través del método transeptos lineales. Para el desplazamiento hasta los sitios de muestreo se utilizó una panga con motor fuera de borda de 75 HP. Se utilizó equipo de buceo para las inmersiones, cinta métrica para delimitar dos transeptos de 30 metros de longitud por 4 metros de ancho, en cada sitio de muestreo. Se utilizó torpedo propulsor para el desplazamiento bajo el agua, video cámaras submarinas de alta resolución para la toma de videos y fotografías, permitiendo la identificación de la flora y fauna presente. La orientación de los transeptos se estableció tomando en cuenta el gradiente de profundidad de más profundo a más somero, hasta llegar a la cresta arrecifal.

La identificación de los corales, octocorales, pastos marinos y las algas, se llevó a nivel de especies, utilizando guía ilustrada de corales del Caribe, Hernández (2009). Como base para determinar el estado de salud de cada uno de los arrecifes coralinos y de manera general el estado de salud de todo el sistema de arrecifes coralinos presentes, se consideró la metodología de (SmithReefs, 2018). Tabla 1. Para ello se determinó la magnitud de cuatro indicadores: cobertura de corales vivos, cobertura de macro algas carnosas, biomasa de peces herbívoros y biomasa de peces comerciales. También se colectó información de la riqueza de especies de fauna invertebrada, tanto de valor comercial como de valor ecológico, entre ellos herbívoros que regulan las poblaciones de algas en los arrecifes, como erizos de mar, estrellas de mar, entre otros.

El análisis se realizó considerando el reporte del arrecife mesoamericano (SmithReefs, 2018), utilizando los rangos y la escala numérica. Los rangos son divididos en: muy bien, bien, regular, mal y crítico. Cada rango se determina de acuerdo al valor numérico obtenido al promediar los valores de los cuatro indicadores y así se obtiene el cálculo del Índice de Salud Arrecifal (ISA), para cada punto de muestreo. Tabla 2.

Tabla 1. Valores de los indicadores

\begin{tabular}{|c|c|c|c|c|}
\hline Rango & $\begin{array}{c}\text { Cobertura de } \\
\text { corales }\end{array}$ & $\begin{array}{c}\text { Cobertura de } \\
\text { macroalgas } \\
\text { carnosas }\end{array}$ & $\begin{array}{c}\text { Biomasa de } \\
\text { peces herbí- } \\
\text { voros }\end{array}$ & $\begin{array}{c}\text { Biomasa de } \\
\text { peces comer- } \\
\text { ciales }\end{array}$ \\
\hline Muy bien & $40 \%$ & $1 \%$ & $3,290 \mathrm{gr}$ & $1620 \mathrm{gr}$ \\
\hline Bien & $20 \%$ & $5 \%$ & $2,740 \mathrm{gr}$ & $1,210 \mathrm{gr}$ \\
\hline Regular & $10 \%$ & $12 \%$ & $1,860 \mathrm{gr}$ & $800 \mathrm{gr}$ \\
\hline mal & $5 \%$ & $25 \%$ & $990 \mathrm{gr}$ & $390 \mathrm{gr}$ \\
\hline crítico & $<5 \%$ & $>25 \%$ & $<990 \mathrm{gr}$ & $<390 \mathrm{gr}$ \\
\hline
\end{tabular}

Tabla 2. Rango y escala

\begin{tabular}{|c|c|}
\hline Rango & Escala \\
\hline Muy Bien & $4.3-5.0$ \\
\hline Bien & $3.5-4.2$ \\
\hline Regular & $2.7-3.4$ \\
\hline Mal & $1.9-2.6$ \\
\hline Critico & $1.0-1.8$ \\
\hline
\end{tabular}




\section{Resultados}

Los arrecifes coralinos en Cayos Miskitos son someros, con profundidad < 30mts. Se encuentran rodeados de extensos lechos de pastos marinos y algas. Se observan arrecifes en parches, colonias de corales aisladas y crestas arrecifales orientadas de Norte a Sur. También se encuentran jardines de octocorales. Al disminuir la profundidad del arrecife, se hacen más abundantes los corales pétreos y disminuyen los octocorales. Los corales más comunes presentes son, el coral de fuego (Millepora complanata), el coral mostaza (Porites astreoides), el coral de estrellas (Siderastrea sidérea) y según llegamos a la cresta arrecifal aumenta la presencia del coral de cuerno de alce (Acropora palmata). La cresta arrecifal se encuentra muy dañada con gran cantidad de corales muertos, pero algunas están siendo colonizadas por otras colonias de corales de la misma o de otra especie. Esta situación es reportada por Fonseca et al (2001), para estos arrecifes de coral.

En los arrecifes coralinos de Cayos Miskitos, Fonseca et al (2001), reporta 39 especies. En el presente estudio se lograron reportar 23 especies de corales marinos, pero cuatro especies son nuevos reportes, por lo que el listado se aumenta a 43 especies de corales (Tabla 3). La densidad promedio de colonias fue de 5.5 colonias/10 m. esto considerando que dos sitios muestreados: Creole Bar y Bojotle Kira, se encuentran cubiertos de pasto de marino en su totalidad. El diámetro y la altura promedio de las colonias coralinas para todo el sistema arrecifal es alta $(59.7 \mathrm{~cm}$ y $46.2 \mathrm{~cm}$ respectivamente). El promedio de blanqueamiento para Cayos Miskitos es de $4 \%$ y el de incidencia de enfermedades es de $3 \%$.

Los octocorales se encuentran aparentemente mejor representados en las zonas de mayor profundidad. La especie en abanico Gorgonia ventalina es la que más abunda. Otros géneros presentes son de formas arbustivas y pluma.

Entre los arrecifes de coral muestreados, uno (Farrel Reef "Miskitu Reef") ubicado al sureste de Cayos Miskitos, resultó en el rango muy bien. 10 arrecifes se encuentran en el rango de bien, tres en el rango de regular y cuatro en el rango de crítico (Tabla 4). En el rango crítico se encuentran los arrecifes: Creole Bar en el Sur, Ryan, Maxide y Bojotle Kira al noreste.

Tabla 3. Especies de corales marinos reportados en los arrecifes de coral de la Reserva Biológica cayos Miskitos

\begin{tabular}{|c|c|c|c|}
\hline \multicolumn{2}{|c|}{ MILLEPORINES. Phylum Cnidaria, Clase Hydrozoa, Orden Milleporina } & Solenastrea bournoni & Coral estrella suave \\
\hline Millepora alcicornis & Coral de fuego delicado & Solenastrea hyades & Coral estrella \\
\hline Millepora complanata & Coral de fuego & Diploria strigosa & Coral cerebro \\
\hline \multicolumn{2}{|c|}{} & Diploria labyrinthiformis & Coral cerebro \\
\hline SCLERACTINIANS. Phylum Cnidaria, Clase Anthozoa, Orden Scleractinia) & Diploria clivosa & Coral cerebro \\
\hline Acropora cervicornis & Coral de cuerno de ciervo & Colpophyllia natans & Coral cerebro gigante \\
\hline Acropora palmata & Coral de cuerno de alce & Meandrina meandrites & Coral laberinto pequeño \\
\hline Acropora prolifera & Coral cuerno de ciervo & Dendrogyra cylindrus & Coral pilar \\
\hline Porites porites & Coral de dedos & Manicina areolata & Coral de rosa \\
\hline Porites divaricata & Porites divaricata & Leptoseris cucullata & Coral lechuga \\
\hline Porites furcata & Porites furcata & Agaricia grahamae & Coral oreja \\
\hline Porites astreoides & Coral mostaza & Agaricia agaricites & Coral lechuga común \\
\hline Oculina diffusa & Coral arbusto de marfil & Agaricia tenuifolia & Coral lechuga \\
\hline Madracis mirabilis & Coral de lápiz & Mycetophyllia danaana & Coral cactus \\
\hline Madracis decactis & Coral de coronillas & Mycetophyllia lamarckiana & Coral hongo común \\
\hline Stephanocoenia michelinii & Coral radiante & Mycetophyllia aliciae & Coral hongo \\
\hline Montastraea annularis & Coral estrella lobulado & Mycetophyllia ferox & Coral cactus aspero \\
\hline Montastraea faveolata & Coral estrella laminar & Isophyllastrea rigida & Coral estrella aspero \\
\hline
\end{tabular}




\begin{tabular}{|c|c|c|c|}
\hline Montastraea franksi & Coral estrella & Scolymia cubensis & Coral de Alcachofa \\
\hline Montastraea cavernosa & Coral estrella gigante & Scolymia lacera & Coral de hongo solitario \\
\hline Dichocoenia stokesii & Coral estrella elíptico & Mussa angulosa & Coral de flores \\
\hline Favia fragum & Coral pelota de golf & Eusmilia fastigiata & Coral de flor \\
\hline Siderastrea siderea & Coral estrella & Undaria tenuifolia & Coral escleractínio \\
\hline Siderastrea radians & Coral estrella pequeño & & \\
\hline
\end{tabular}

\section{Algas}

Las algas se encuentran en todos los estratos de los arrecifes coralinos en los Cayos Miskitos. Los sitios con alta cobertura de algas carnosas, que presentan estado crítico de este indicador son: London Reef al sureste, Ahuya Luphia al noreste y Ryan al noroeste. Además, cinco arrecifes coralinos presentan mala condición de cobertura de algas carnosas: Macandra al noreste, Toro Cay al Oeste, San-San Reef y Northwest Sport (Witties) al sureste y Yanka Laya en el noroeste. Tabla 4. Este resultado indica una alta colonización y amenaza para los arrecifes de coral por las algas carnosas.

Las algas carnosas registran un promedio de altura de $7 \mathrm{~cm}$, este es superior comparado con la altura promedio de las algas calcáreas con $2.5 \mathrm{~cm}$ y las coralinas con $1 \mathrm{~cm}$. El rango de alturas con mayor variación se dio dentro de las carnosas, Bojotle kira obtuvo el mayor dato con $10.47 \mathrm{~cm}$ y el menor fue en Macandra, $2.40 \mathrm{~cm}$.

\section{Peces herbívoros}

Los peces herbívoros se encuentran en todos los arrecifes coralinos. Si bien la biomasa varía de un arrecife a otro, se puede observar que estas especies se están aumentando, dado que más del 80\% de los peces observados eran juveniles. Las especies más abundantes son pez loro y pez loro rallado (Scarus taeniopterus y Scarus iseri). Estas especies tienen una población buena y estable en los arrecifes y pueden llegar a mantener manejo del control en la población de algas. En el 50 \%, nueve arrecifes muestreados la población de peces herbívoros resultó en el rango muy bien, uno en el rango bien, cinco en el rango mal y tres en el rango crítico, los cuales son: Creol Bar en el Sur, Maxide y Bojotle Kira al noreste. Tabla 4. Cabe mencionar que estos tres arrecifes en condición crítica de peces carnívoros también presentan condición crítica de arrecifes de coral, indicando alta dependencia entre ellos.

Otras especies herbívoras de importancia en el control de la población de algas son los invertebrados erizo de mar (Diadema antillarum) y estrella de mar (Oreaster reticulatus). Estos organismos se encuentran en los arrecifes de coral y en los pastos marinos.

\section{Peces de importancia comercial}

Los peces de importancia comercial se encuentran en todos los arrecifes coralinos. Igualmente, la biomasa varía de un arrecife a otro. Las especies mejor representadas son barracuda (Sphyraena barracuda) y tiburón nodriza (Ginglymostoma cirratum). Además, pargos en estado juvenil. En el $50 \%$, (nueve) de los arrecifes muestreados la población de peces de importancia comercial resultó en el rango muy bien, tres en el rango bien, dos en el rango regular, uno en el rango mal y tres en el rango crítico, los cuales son: Ahuya Lupia, Maxide y Bojotle Kira al noreste. Tabla 4.

Los arrecifes de coral mantienen la estabilidad ecológica, donde se desarrollan los peces de valor comercial que mantienen la economía de las comunidades indígenas miskitas. Los arrecifes de coral son importantes sitios de alimentación para los tiburones nodrizas, según Infotiburones 2018, allí se alimentan los crustáceos, langostas, camarones, además de pequeños peces, corales marinos y conchos. 
Tabla 4. Cobertura Indicadores: corales vivos, de macro algas carnosas, biomasa de peces herbívoros y peces comerciales para cada 100 metros cuadrados de áreas en los 18 arrecifes estudiados en Cayos Miskitos.2018.

\begin{tabular}{|c|c|c|c|c|c|}
\hline Sitio & $\begin{array}{c}\text { Cobertura de } \\
\text { Coral }\end{array}$ & $\begin{array}{l}\text { Cobertura de } \\
\text { Macroalgas }\end{array}$ & Peces Herbívoro & Peces Comercial & ISA \\
\hline Lamarka Reef & 4 & 4 & 5 & 5 & 4.5 \\
\hline Dump & 4 & 4 & 5 & 4 & 4.25 \\
\hline Ned Thomas Cay & 4 & 3 & 5 & 5 & 4.25 \\
\hline Nasa Cays (Nasa Reef) & 3 & 4 & 5 & 5 & 4.25 \\
\hline Hamkira & 4 & 3 & 5 & 5 & 4.25 \\
\hline Macandra & 3 & 2 & 5 & 5 & 3.75 \\
\hline Farrel Reef (Miskitu Reef) & 5 & 3 & 2 & 5 & 3.75 \\
\hline Wiplyn & 4 & 4 & 2 & 5 & 3.75 \\
\hline Sam-Sam Reef (Franklin Reef) & 3 & 2 & 5 & 4 & 3.5 \\
\hline London Reef & 4 & 1 & 2 & 5 & 3 \\
\hline Toro Cay & 4 & 2 & 3 & 3 & 3 \\
\hline Ahuya Luphia & 4 & 1 & 5 & 1 & 2.75 \\
\hline Creole Bar & 1 & 4 & 1 & 5 & 2.75 \\
\hline Northwest Spot (Witties) & 4 & 2 & 2 & 3 & 2.75 \\
\hline Ryan & 1 & 1 & 5 & 4 & 2.75 \\
\hline Yanka Laya & 4 & 2 & 2 & 2 & 2.5 \\
\hline Maxide & 1 & 4 & 1 & 1 & 1.75 \\
\hline Bojotle Kira & 1 & 4 & 1 & 1 & 1.75 \\
\hline
\end{tabular}

\section{Indice de salud de arrecifal en la Reserva Biológica Cayos Miskitos}

En base a estos resultados del análisis de los indicadores se logró obtener el Índice de Salud Arrecifal para cada uno de los sitios muestreados y en su conjunto para los Cayos Miskitos. De los 18 arrecifes muestreados el 6\% (1) se encuentra en el rango de muy bien y el $44 \%$ (8) se encuentran el rango de bien, estas dos categorías suman el 50\% de los arrecifes. En la categoría regular se encuentra el 33\% (6), mientras en las categorías mal el 6\% (1) y crítico 11\% (2). Gráfico 1.

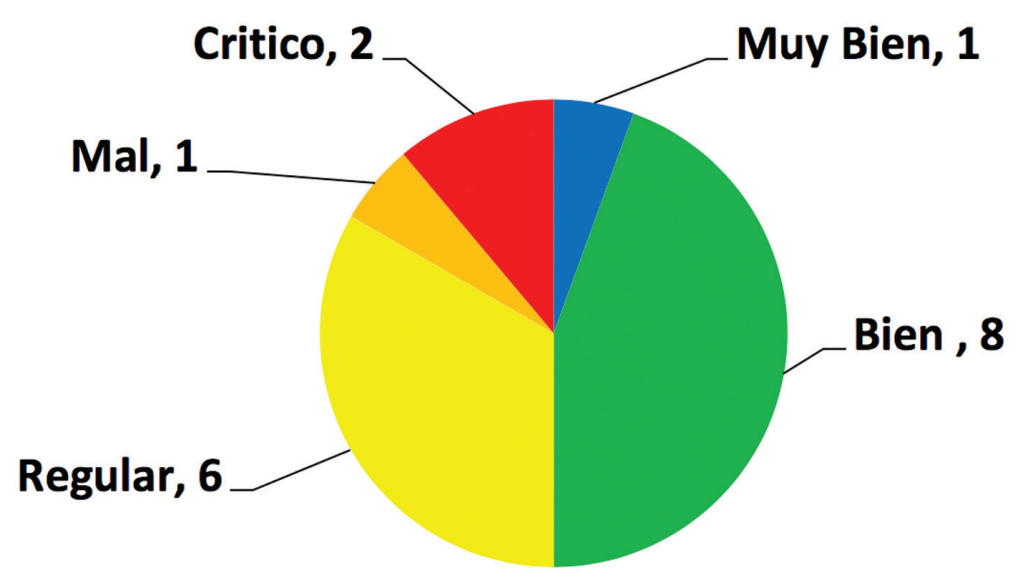

Gráfico 1. Número de arrecifes ubicados en cada rango, según resultado de indicadores. Cayos Miskitos. 
De acuerdo al análisis de los indicadores en cada uno de los 18 arrecifes de coral y de manera general al promediar los datos de cada indicador, se obtuvo que la cobertura de corales y de macro algas carnosas se ubican en el rango de regular, mientras que biomasa de peces herbívoros y biomasa de peces comerciales se ubican en el rango bien. Al promediar todos los resultados se obtuvo el valor del Índice de Salud Arrecifal para Cayos Miskitos, con un valor de 3.29, situándose en el rango de regular (Gráfico 2). Este valor es superior a lo reportado en el arrecife mesoamericano, donde se presenta un promedio de 2.8 (SmithReefs, 2018).

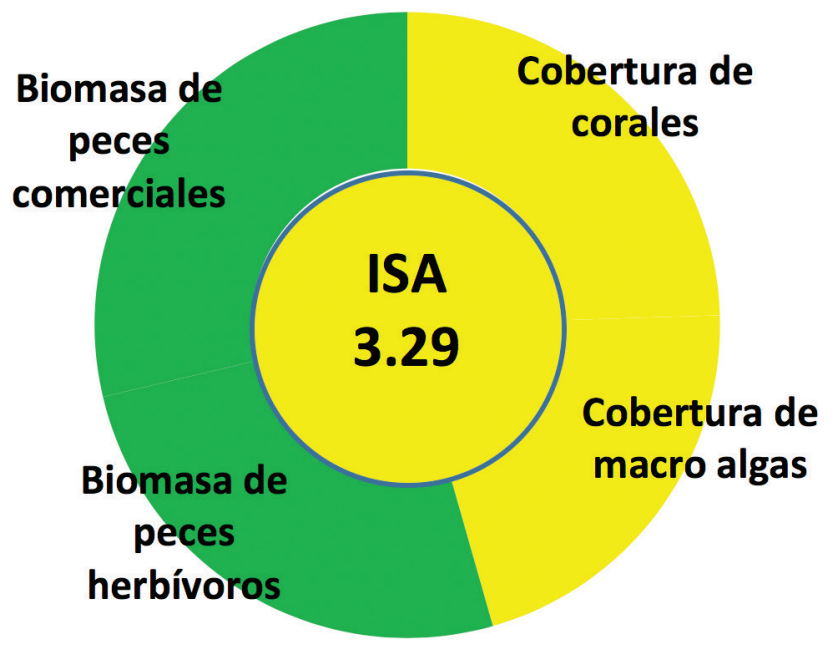

Gráfico 2. Indice de Salud Arrecifal.

\section{Conclusiones}

Los arrecifes coralinos en Cayos Miskitos son someros con profundidades <30 metros. Los principales arrecifes se encuentran en profundidades de o a 5 metros, rodeados de pasto marino.

El promedio de blanqueamiento es de $4 \%$ y el de incidencia de enfermedades es de $3 \%$. Estos valores son similares a lo encontrado en arrecifes someros de México, Belice y Honduras, (Kramer y Kramer 2000).

Las crestas arrecifales se encuentran muy dañadas por incidencias climáticas. La incidencia directa más reciente se dio con paso del huracán Félix en el año 2007. La fuerza del sistema, generó fuertes oleajes y movimientos de masas de agua lo que llego a remover y levantar del suelo a colonias de corales creando promontorios de especímenes muertos.

Los corales muertos que se encuentran en los arrecifes coralinos, están sirviendo como sustrato donde se están estableciendo nuevas colonias de corales constructores.

En todos los arrecifes coralinos de Cayos Miskitos se presenta incidencia de sedimentos suspendidos, siendo más evidente al noreste. Esta sedimentación podría estar aportando nutrientes que favorecen la proliferación de algas carnosas.

Los indicadores de salud arrecifal en cayos miskitos presentaron buena presencia, indicando que estos arrecifes están cumpliendo su función de protección y estabilización ecológica, brindando refugio a especies que sirven de base para la alimentación y la economía de las poblaciones indígenas costeras del Caribe Norte de Nicaragua. 
El estado de salud del sistema arrecifal en Cayos Miskitos se encuentra en el rango de regular con una escala de 3.29, superando la escala del arrecife mesoamericano que promedia 2.8 .

El repoblamiento de los arrecifes coralinos que se encuentran en rango crítico (Creole Bar, Ryan, Maxide y Bojotle Kira), podrían incentivarse, mediante el establecimiento de técnicas artificiales, con métodos asexuales (replantación de trozos prendedizos) y construcción de fincas de arrecifes. Mercado et al 2013, Ruiz et al (2012).

\section{Agradecimiento}

Esta publicación obtuvo el financiamiento del Proyecto Ecosistemas Costeros, auspiciado por: Unión Europea, Dka Austria, Cooperación Austriaca para el Desarrollo y Horizontzooo.

\section{Lista de referencias}

Alevizon, W. 1993. A preliminary survey of the coral reefs and reef fisheries of the Miskitu cays, Nicaragua. CCC, Florida. 16 p.

Cortés, J. 1997. Status of the Caribbean coral reef of Central America. Proc. 8th Int. Coral Reefs Sym. 1: 335-340.

Fonseca A, Jadder Mendoza, Luisa Paredes, Odalisca Breedy, Pedro Mercado, (2001). Evaluación ecológica rápida del sistema arrecifal de cayos miskitus. WWF-PROARCA COSTAS/URACCAN-IREMADES.

Hernández, Edwin. Guía para la identificación de los corales más comunes en Puerto Rico. Departamento de recursos naturales y ambientales. Programa de manejo de zonas costaneras.

Jameson, S.C. 1996. Coral reef ecosystem survey and management recommendations. National Oceanic and Atmospheric Administration. USAID. 18 p.

Kramer, P.A. \& P.R. Kramer. 2000. Ecological status of the Mesoamerican Barrier Reef System. Impacts of Hurricane Mitch and 1998 coral bleaching. RSMAS/World Bank. 73 p.

MARENA 1999. Biodiversidad en Nicaragua: Un estudio de país. MARENA, PANIF. 1ra edición. 469 p.

Marshall, M. 1994. Seagrasses of the Miskito Cays Biological Reserve. Preliminary report to USAID. 16 p.

Mercado, A., Edwin A. Hernández Delgado, José E. Rivera Rivera, Mayra Rivera Rivera, Samuel E. Suleimán Ramos, Iván Olivo Maldonado, Jaime S. Fonseca Miranda, Evelyn A. Rodríguez Inoa. 2013. Protocolo para la propagación y la restauración de poblaciones del coral cuerno de ciervo, acropora cervicornis: estrategias de bajo costo de la sociedad ambiente marino. Centro para la Ecología Tropical Aplicada y Conservación (CATEC) de la Universidad de Puerto Rico, Recinto de Río Piedras HRD \#0734826.

Ogden, J.C. \& E.H. Gladfelter. 1983. Coral reefs, seagrass beds and mangroves: their interaction in coastal zones of the Caribbean. UNESCO Reports in Marine Science $\mathrm{N}^{\circ} 23$.

Phillips, R.C., R.L. Vadas \& N. Ogden. 1982. The marine algae and seagrasses of the Miskito Bank, Nicaragua. Aquat. Bot. 13: 187-195.

Ruiz, H, Antonio L. Ortiz, Michael Nemeth, Michelle Sch冈rer y Sean Griffin, 2012. Casos de estudio en la restauración de arrecifes de coral de Puerto Rico. 